\title{
Natural Frequency Numerical Solution to an Axially Moving Timoshenko \\ Beam on Fixed Supports
}

\author{
Zhigang Yang ${ }^{1 \mathrm{a}}$, Wanzhen $_{\mathrm{Li}}{ }^{1 \mathrm{~b}}$, Zhenxing Yang $^{1 \mathrm{c}}$,Jiguang Zhang ${ }^{1 \mathrm{~d}}$ \\ 1Department of Architectural Engineering, Rizhao Polytechnic, Rizhao276800, China \\ ayzg5151@163.com, ${ }^{b} 305165526 @ q q . c o m,{ }^{\circ} 346561875 @ q q . c o m,{ }^{d} 303655380 @ q q . c o m$
}

\begin{abstract}
KEYWORDS: Timoshenko model; axially moving beams; natural frequencies; Galerkin method; differential quadrature method

ABSTRACT: In this paper, natural frequencies of axially moving beams on fixed supports are analyzed based on Timoshenko model by performing the Galerkin method and the differential quadrature method. Some numerical examples are presented to demonstrate the effects of related parameters on the frequencies, such as the axial speed and the stiffness. The results obtained by the differential quadrature method and the complex mode approach have a good agreement; while the results obtained with the Galerkin method are a little different.
\end{abstract}

The axially moving records Continuum model are widely used in research, manufacture and production area of military, aerospace and mechatronic engineering. Such like aerial cable tramways, power transmission belt, band saws and lift cable etc. The research of axially moving beams transverse vibration and control has important value of application and practical.

Euler beams model is kind of classical model that simplify and efficacious. Öz[1], Feng [2], Chen et al. [3],Zhang [4],Chen[5] all have research in vibration problem of axially moving Euler beams. But for the slender beams, the computed results of Euler beams model can not equal Timoshenko model in accuracy. Yang used the complex modal analysis and Galerkin method to solution the natural frequency of axially moving Timoshenko beams, analysis the influence of the natural frequency precision by complex modal analysis method、2-trem Galerkin truncation and 4-trem Galerkin truncation method. Ghayesh and Balar[7] researched the axially moving beams transverse vibration natural frequency under clamped-clamped boundary conditions by Semi-analytical and semi-numerical method. Li [8] and others researched the axially moving Timoshenko beams natural frequency under simple support with torsion springs by Semi-analytical and semi-numerical method. Tang [9] and others researched the Timoshenko mode-1 axially moving beams natural frequency under all kinds of boundary conditions by complex modal analysis method. Ding [11] and others researched the vibrations under simply support conditions through finite difference method. This paper used the Galerkin truncation method and DQM to research axially moving beams Timoshenko beams natural frequency under clamped-clamped boundary conditions, and compared the numerical result with the result of complex modal analysis method.

\section{THE GOVERNING EQUATION}

The zero dimension form governing equation of axially moving Timoshenko beams transverse vibration is:

$y_{t_{t}}+2 v y,_{x t}+\left(v^{2}-1\right) y,_{x x}-\left(k_{1}+k_{2}+k_{3}-k_{2} v^{2}\right) y,_{x x t t}+\left(k_{1}+k_{4}-k_{1} v^{2}\right) y,_{x x x x}$

$+k_{2}\left(y_{\text {tttt }}+2 v y_{\text {xttt }}\right)-2 k_{1} v y$, xxxt $=0$ 
The $y$ is lateral displacement of moving beams, $x$ is vertical displacement of beams, $t$ is characterization time, $v$ is characterization transverse velocity, $k_{1}$ and $k_{2}$ are characterization shear modulus, $k_{3}$ is characterization intersection angle, $k_{4}$ is characterization flexural rigidity.

The axially moving beams transverse vibration natural frequency under clamped-clamped boundary conditions

$y(0, t)=y(1, t)=0, y,_{x}(0, t)=y,_{x}(1, t)=0$

\section{NUMERICAL METHOD}

The following will use two kinds of methods for solving system.

\section{Galerkin method}

The continuum equation (1) of motion of a simply supported timoshenko beam can be cast in the standard form of ordinary differential equations via the method of Galerkin. Suppose $\Phi_{i}(x)$ can be approximated by

$\Phi_{i}(x)=\cos \psi_{i} x-\cosh \psi_{i} x-\frac{\cos \psi_{i}-\cosh \psi_{i}}{\sin \psi_{i}-\sinh \psi_{i}}\left(\sin \psi_{i} x-\sinh \psi_{i} x\right)$

Where $\psi_{i}$ satisfy $\cos \psi_{i} \cosh \psi_{i}-1=0$.

The solution equation (1) may be expanded into the series of eigenfunctions of the stationary beam with two fixed ends $y(x, t)=\Phi^{\mathrm{T}} \mathrm{q}$, where the infinite column matrixes $\mathbf{q}(t)$ and $\Phi(x)$ are respectively assembled by the generalized displacements and the stationary eigenfunctions, namely $\mathbf{q}=\left[\mathrm{q}_{1}(\mathrm{t}), \mathrm{q}_{2}(\mathrm{t}), \ldots, \mathrm{q}_{\mathrm{N}}(\mathrm{t})\right]^{\mathrm{T}}, \boldsymbol{\Phi}=\left[\Phi_{1}(\mathrm{t}), \Phi_{2}(\mathrm{t}), \ldots, \Phi_{\mathrm{N}}(\mathrm{t})\right]^{\mathrm{T}}$. Substituting matrixes $\mathbf{q}(t)$ and $\boldsymbol{\Phi}(x)$ into equation (1), multiplying the resulting equation by $\Phi(x)$ and integrating the product from 0 to 1 , $\ddot{\mathbf{q}}+2 v \mathbf{B}_{1} \dot{\mathbf{q}}+\left(v^{2}-1\right) \mathbf{B}_{2} \mathbf{q}-\left(k_{1}+k_{2}+k_{3}-k_{2} v^{2}\right) \mathbf{B}_{2} \ddot{\mathbf{q}}+\left(k_{1}+k_{4}-k_{1} v^{2}\right) \mathbf{B}_{4} \mathbf{q}+$

$k_{2}\left(\mathbf{q}^{(4)}+2 v \mathbf{B} \dddot{\mathbf{q}}\right)-2 k_{1} v \mathbf{B} \mathbf{3} \dot{\mathbf{q}}=0$

where

$\mathbf{B}_{1}=\frac{\int_{0}^{1} \boldsymbol{\Phi} \boldsymbol{\Phi}^{\prime \mathrm{T}} \mathrm{d} x}{\int_{0}^{1} \boldsymbol{\Phi} \boldsymbol{\Phi}^{\mathrm{T}} \mathrm{d} x}, \mathbf{B}_{2}=\frac{\int_{0}^{1} \boldsymbol{\Phi} \boldsymbol{\Phi}^{\prime \prime \mathrm{T}} \mathrm{d} x}{\int_{0}^{1} \boldsymbol{\Phi} \boldsymbol{\Phi}^{\mathrm{T}} \mathrm{d} x}, \mathbf{B}_{3}=\frac{\int_{0}^{1} \boldsymbol{\Phi} \boldsymbol{\Phi}^{\prime \prime \prime \mathrm{T}} \mathrm{d} x}{\int_{0}^{1} \boldsymbol{\Phi} \boldsymbol{\Phi}^{\mathrm{T}} \mathrm{d} x}, \mathbf{B}_{4}=\frac{\int_{0}^{1} \boldsymbol{\Phi} \boldsymbol{\Phi}^{\prime \prime \prime \mathrm{T}} \mathrm{d} x}{\int_{0}^{1} \boldsymbol{\Phi} \boldsymbol{\Phi}^{\mathrm{T}} \mathrm{d} x}$

Turn (4) into the matrix form

$$
\dot{\mathbf{z}}=\mathbf{A z}
$$

Where $\mathbf{z}=\left(\begin{array}{llll}\mathbf{q} & \dot{\mathbf{q}} & \ddot{\mathbf{q}} & \dddot{\mathbf{q}}\end{array}\right)^{\mathrm{T}}$,

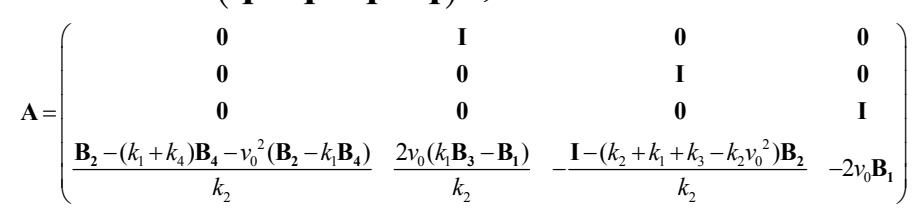

The $\mathbf{0}$ and I mean zero matrix and unit matrix respectively in $\mathbf{N} \times \mathbf{N}$. A is gyroscopic matrix, all characteristic value of $\mathbf{A}$ is paired imaginary number, and it can change matrix into

$$
\mathbf{T}^{-1} \mathbf{A} \mathbf{T}=\left(\begin{array}{llll}
\omega_{1} \mathbf{J} & & & \\
& \omega_{2} \mathbf{J} & & \\
& & \ddots & \\
& & & \omega_{2 N} \mathbf{J}
\end{array}\right) \mathbf{J}=\left(\begin{array}{cc}
0 & -1 \\
1 & 0
\end{array}\right)
$$

\section{Differential Quadrature Method}

Assumption the solution formula of equation (1) is

$$
y(x, t)=\psi(x) e^{\lambda t}
$$

The $\psi(x)$ intrinsic mode function, $\lambda$ is Complex Eigenvalue 
Substitute (9) into (1) and (2) can obtained respectively

$$
\begin{gathered}
k_{2} \lambda^{4} \psi+2 k_{2} v \lambda^{3} \psi^{\prime}+\lambda^{2} \psi-\left(k_{1}+k_{2}+k_{3}-k_{2} v^{2}\right) \psi^{\prime \prime} \lambda^{2} \\
+2 v \psi^{\prime} \lambda-2 k_{1} \nu \psi^{\prime \prime \prime} \lambda+\left(v^{2}-1\right) \psi^{\prime \prime}+\left(k_{1}+k_{4}-k_{1} v^{2}\right) \psi^{\prime \prime \prime \prime}=0 \\
\psi(0)=\psi(1)=0 ; \psi^{\prime}(0)=\psi^{\prime}(1)=0
\end{gathered}
$$

Use the $\delta$-technique deal with clamped-clamped boundary conditions (2), The number of the sampling point is $N$ in the $\mathrm{x}$ direction. Introduce the $N$ sampling point as $x_{1}=0, x_{2}=\delta, x_{N-1}=1-\delta, x_{N}=1, x_{i}=\frac{1}{2}\left[1-\cos \frac{(i-1) \pi}{N-1}\right] \cdot(i=3,4, \ldots, N-2)$

Based on differential quadrature rules

$$
f_{x}^{(s)}\left(x_{i}\right)=\sum_{j=1}^{N} A_{i j}^{(s)} f_{j} \quad(i=1,2, \ldots, N)
$$

$\psi,_{x}\left(x_{i}\right)=\sum_{j=1}^{N} A_{i j}^{(1)} \psi_{j}, \psi,_{x x}\left(x_{i}\right)=\sum_{j=1}^{N} A_{i j}^{(2)} \psi_{j}, \psi,_{x x x}\left(x_{i}\right)=\sum_{j=1}^{N} A_{i j}^{(3)} \psi_{j}, \psi,,_{x x x x}\left(x_{i}\right)=\sum_{j=1}^{N} A_{i j}^{(4)} \psi_{j}$

where the weight coefficient is defined by

$$
A_{i j}^{(1)}=\left\{\begin{array}{lr}
\prod_{\mu=1, \mu \neq i}^{N}\left(x_{i}-x_{\mu}\right) /\left[\left(x_{i}-x_{j}\right) \prod_{\mu=1, \mu \neq j}^{N}\left(x_{j}-x_{\mu}\right)\right] & (i, j=1,2, \cdots, N, i \neq j) \\
\sum_{\mu=1, \mu \neq i}^{N} \frac{1}{x_{i}-x_{\mu}} & (i=1,2, \cdots, N, i=j)
\end{array}\right.
$$

and in the case of $r=2,3, \ldots, N-1$

$$
A_{i j}^{(r)}=\left\{\begin{array}{lr}
r\left(A_{i i}^{(r-1)} A_{i j}^{(1)}-\frac{A_{i j}^{(r-1)}}{x_{i}-x_{j}}\right) & (i, j=1,2, \cdots, N, i \neq j) \\
-\sum_{\mu=1, \mu \neq i}^{N} A_{i \mu}^{(r)} & (i=1,2, \cdots, N, i=j)
\end{array}\right.
$$

The $A_{i j}^{(r)}(i, j=1,2, \cdots, N)$ mean that the condition [10] $A(r)=A(1) A(r-1)$.

Following the above definitions, the differential quadrature scheme of the linear generating system may be obtained as

$$
\begin{aligned}
& k_{2} \psi_{i} \lambda^{4}+2 v k_{2} \lambda^{2} \sum_{j=1}^{N} A_{i j}^{(1)} \psi_{j}+\lambda^{2} \psi_{i}-\left(k_{1}+k_{2}+k_{3}-k_{2} v^{2}\right) \lambda^{2} \sum_{j=1}^{N} A_{i j}^{(2)} \psi_{j} \\
& +2 v \lambda\left(\sum_{j=1}^{N} A_{i j}^{(1)} \psi_{j}-k_{1} \sum_{j=1}^{N} A_{i j}^{(3)} \psi_{j}\right)+\left(v^{2}-1\right) \sum_{j=1}^{N} A_{i j}^{(2)} \psi_{j}+\left(k_{1}+k_{4}-k_{1} v^{2}\right) \sum_{j=1}^{N} A_{i j}^{(4)} \psi_{j}=0 \\
& i=3, \ldots, N-2 \\
& \psi(1)=\psi(N)=0 ; \sum_{j=1}^{N} A_{2 j}^{(1)} \psi_{j}=\sum_{j=1}^{N} A_{(N-1) j}^{(1)} \psi_{j}=0
\end{aligned}
$$

Eq. (17) can be rewritten into the matrix form,

$$
\left(\lambda^{4} \mathbf{H}+\lambda^{3} \mathbf{F}+\lambda^{2} \mathbf{M}+\lambda \mathbf{G}+\mathbf{K}\right)\{\boldsymbol{\psi}\}=0
$$

Where $\mathbf{H}, \mathbf{F}, \mathbf{M}, \mathbf{G}, \mathbf{K}$ are matrix of order $N \times N .\{\boldsymbol{\psi}\}$ denotes the generalized displacements. It is of order $N \times 1, N=11$.

\section{RESULT AND ANALYSIS}

Apply Galerkin method, we can get $n$-th natural frequency of axially moving beams in (11), we can get $n$-th natural frequency of axially moving beams by DMQ in (19). The solutions of complex modal analysis method can be used to exact solution without consider exact solution. But Galerkin 
method leads to error inevitable for truncation method. The figure 1 and figure 2 both provide the situation of Timoshenko model moving beams first two-step natural frequency changed following axially moving speed in difference stiffness coefficient under clamped-clamped boundary conditions. Physical parameter of Timoshenko beams are $P=107 \mathrm{~N}, A=9.0 \times 10^{-3} \mathrm{~m}^{2}, E=1.69 \times 10^{11} \mathrm{~Pa}, k=5 / 6, l=0.3 \mathrm{~m}, \mathrm{G}=6.6 \times 1010 \mathrm{~Pa} \quad ; \quad$ the $\quad$ corresponding non-quantity parameters are $k_{1}=9.0 \times 10^{-3}, k_{2}=5.90 \times 10^{-5}, k_{3}=4.2 \times 10^{-3}, k_{4}=0.64$. The figure 1 shows the situation of the first two-step natural frequency of system changed with axial velocity by use 4-trem Galerkin method, the figure 2 shows the situation of the first two-step natural frequency of system changed with axial velocity by use DQM.

From figure 1 and figure 2 we can figure out that the first two-step natural frequency of system by use Galerkin method and DQM will increase following stiffness coefficient increase when the axial velocity the same, but when stiffness coefficient is same, the natural frequency of system will decrease following axial velocity increase.

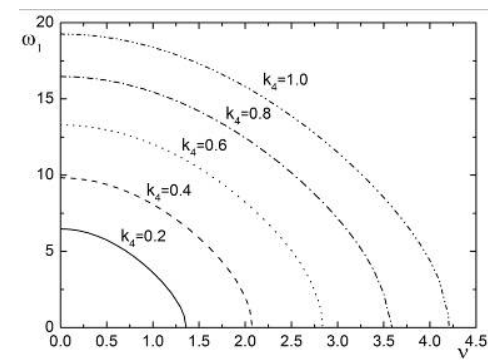

(a) First natural frequency

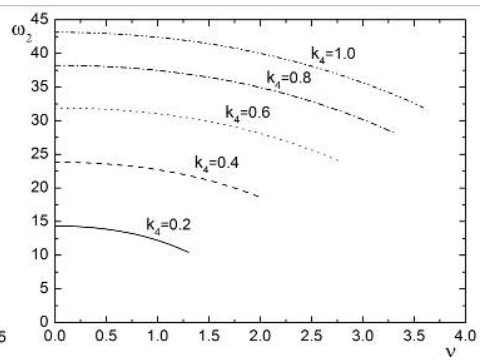

(b) Second natural frequency

Fig.1 Natural frequencies changing with axial speed and constraint stiffness (Galerkin)

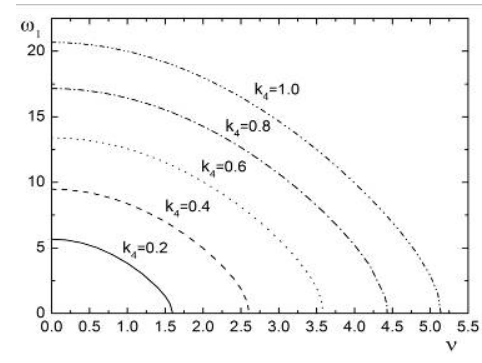

(a) First natural frequency

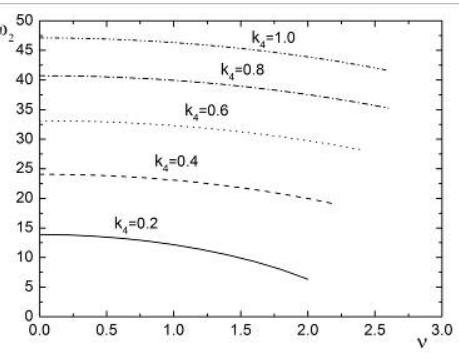

(b) Second natural frequency

Fig. 2 Natural frequencies changing with axial speed and constraint stiffness (DQM)

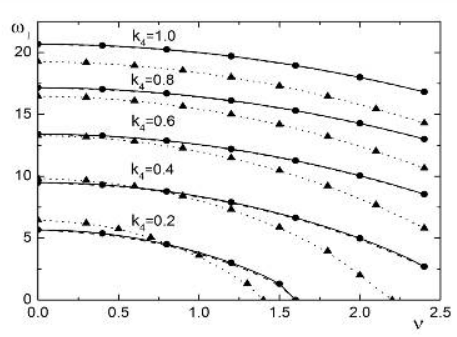

(a) First natural frequency

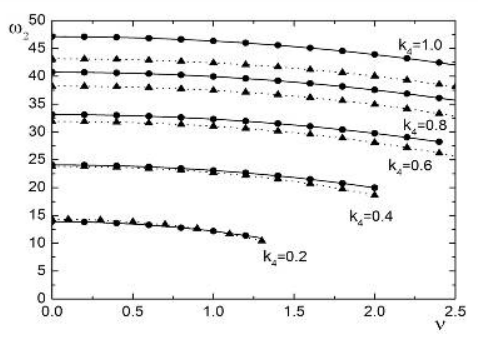

(b) Second natural frequency

Fig. 3 The comparison for the results of above three methods

The figure 3 provides the compare the results of natural frequency by application Galerkin method, DQM with complex modal analysis method. The full lines means first two order natural frequency of system changed with the situation of axial velocity by take advantage of complex modal analysis method, the triangle point's dotted lines represent use 4-trem Galerkin method, the dot dotted lines stand for application DQM method. From the figure we can found this two methods that the result of DQM and complex modal analysis can match well. The results of 4-trem Galerkin truncation method have some difference with the results of DQM method and complex modal analysis method, the three results are similar when stiffness coefficient is small, but when the stiffness coefficient and 
speed are increase, the different of results are more and more bigger. This difference should cause by trial function of truncation. The natural frequency of Timoshenko axially moving beams calculated by Galerkin method and complex modal analysis method under fixed supports will be found.The results obtained by the differential quadrature method and the complex mode approach have a good agreement, while the results obtained with the Galerkin method are a little different.

\section{CONCLUSIONS}

The research of this paper is Timoshenko transverse vibration of axially moving beams under clamped-clamped boundary conditions, take advantage of Galerkin method and DQM respectively to figure up the first two order natural frequency of system changed with the situation of stiffness coefficient and axial velocity, and compare the result of this two method with complex modal analysis method, find out the result of DQM and complex modal analysis this two method can match good. Though the results of 4-term Galerkin truncation method have the same trend between the first two method, they also have a certain difference.

\section{REFERENCES}

[1] Öz H R. On the vibrations of an axially traveling beam on fixed supports with variable velocity [J]. Journal of Sound Vibration, 2001, 239:556-564.

[2] Feng Zhihua, Hu Haiyan. Dynamic stability of a slender beam with internal resonance under a large linear motion [J]. Journal of Mechanics, 2002, 34: 389 -400.

[3] Chen Shuhui, Huang Jianliang. On internal resonance of nonlinear vibration of axially moving beams [J]. Journal of Mechanics, 2005, 37:57-63.

[4] Zhang Wei, Wen Hongbo, Yao Minghui. Periodic and chaotic oscillation of a parametrically excited viscoelastic moving belt with 1:3 internal resonance [J]. Journal of Mechanics, 2004, 36:443-454.

[5] Chen L Q, Wang B.Stability of axially accelerating viscoelastic beams: asymptotic perturbation analysis and differential quadrature validation[J]. European Journal of Mechanics A/Solids, 2009, 28:786-791.

[6] ang XiaoDong, TANG YouQi, Ge XinSheng. Natural frequency analysis of axially moving simply supported Timoshenko beam [J]. Mechanical Strength,2009,31 (2):208-210.

[7] Ghayesh M H, Balar S.Non-linear parametric vibration and stability analysis for two dynamic models of axially moving Timoshenko beams [J]. Applied Mathematical Modelling, 34 (2010):2850-2859.

[8] Biao Li, Hu Ding ,Liqun Chen. Transverse vibration of an axially moving Timoshenko beam with unsymmetrical hybrid supports[J]. Acta Mechanica Solida Sinica,2009, 30(6):565-570.

[9] Tang Y Q, Chen L Q, Yang X D.Natural frequencies, modes and critical speeds of axially moving Timoshenko beams with different boundary conditions. International Journal of Mechanical Sciences.2008, 50, 1448-1458.

[10] Malink M, Bert C W. Implementing multiple boundary conditions in the DQ solution of higher-order PDE's: application to free vibration of plates [J], International Journal for Numerical Methods in Engineering.1996, 39:1237-1258.

[11] Ding Hu, Liqun Chen. Coupled forced response of nonlinear axially moving Viscoelastic Beam [J]. Journal of Shanghai University: JCR Science Edition, 2009, 15(6):649-652. 